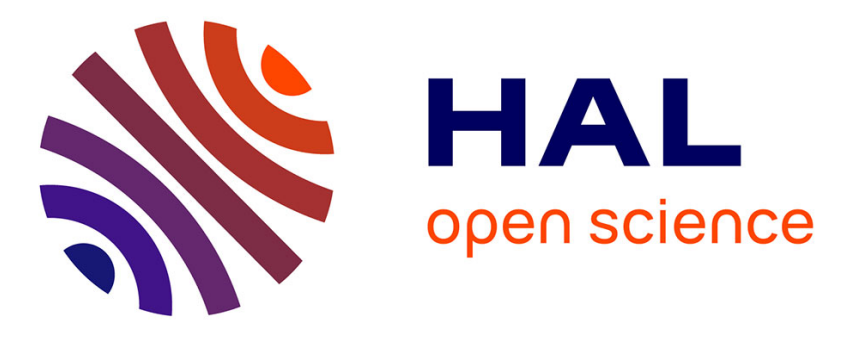

\title{
HF radar antenna near field assessment using a UAV
} Quentin Herbette, Stéphane Saillant, Michel Menelle, Bruno Urbani, Gilbert Auffray, Nicolas Bourey, Muriel Darces, Marc Hélier

\section{To cite this version:}

Quentin Herbette, Stéphane Saillant, Michel Menelle, Bruno Urbani, Gilbert Auffray, et al.. HF radar antenna near field assessment using a UAV. IEEE International Conference on Radar RADAR 2019, Sep 2019, TOULON, France. pp.1-6, 10.1109/RADAR41533.2019.171336 . hal-03306719

\section{HAL Id: hal-03306719 https://hal.science/hal-03306719}

Submitted on 29 Jul 2021

HAL is a multi-disciplinary open access archive for the deposit and dissemination of scientific research documents, whether they are published or not. The documents may come from teaching and research institutions in France or abroad, or from public or private research centers.
L'archive ouverte pluridisciplinaire HAL, est destinée au dépôt et à la diffusion de documents scientifiques de niveau recherche, publiés ou non, émanant des établissements d'enseignement et de recherche français ou étrangers, des laboratoires publics ou privés. 


\section{HF Radar antenna near field assessment using a UAV}

\author{
Quentin HERBETTE, Stéphane SAILLANT, \\ Michel MENELLE, Bruno URBANI, Gilbert AUFFRAY \\ DEMR, ONERA, Université Paris Saclay \\ F-91123 Palaiseau, France \\ quentin.herbette@onera.fr
}

\author{
Nicolas BOUREY, Muriel DARCES, \\ Marc HÉLIER \\ Sorbonne Université \\ Laboratoire d'Électronique et Électromagnétisme, L2E \\ F-75005 Paris, France
}

\begin{abstract}
This paper deals with a system aboard a UAV used to map the electric near field, in amplitude and phase, radiated by an antenna operating in the $\mathrm{HF}$ band (3-30 MHz). The objective is to characterize the radiation pattern of antennas that are commonly integrated in HF surface wave radar. An innovative and original approach is required because usual characterization methods do not suit well for that frequency band. The proposed device is described in the paper and associated constraints are highlighted. A trial of that system has been performed on a monopole antenna, and comparisons between measurements and simulations are in good agreement.
\end{abstract}

Keywords- Unmanned Aerial Vehicle, Near Field, HF Surface Wave Radar

\section{INTRODUCTION}

ONERA is currently deploying biconical antennas for its sky wave and surface wave radars. A photography of a part of the transmitting array of a HF radar operated by ONERA, composed of numerous biconical antennas, is shown below in Fig.1. The large size of HF antennas, and difficulties to model the environment in which they operate, complicate the applications of conventional antenna characterization techniques, as anechoic chamber or Open Area Test Site. Even if HF antenna far field characterization by means of a system aboard a helicopter has already been achieved, that kind of tests are very expensive and difficult to perform [1]. To address this problem, a new method and a new airlifted system are proposed. It will allow to obtain the radiation pattern of an $\mathrm{HF}$ antenna by means of a near-field to far-field transformation, thanks to a previously developed software [2]. One of the features of that software is its ability to take the surface wave radiation into account, which is required to characterize HF antennas, used for surface wave radar, in their own environment. In the future, as the proposed device is flexible, it will also be applied to new innovative transmission systems that are still in development.

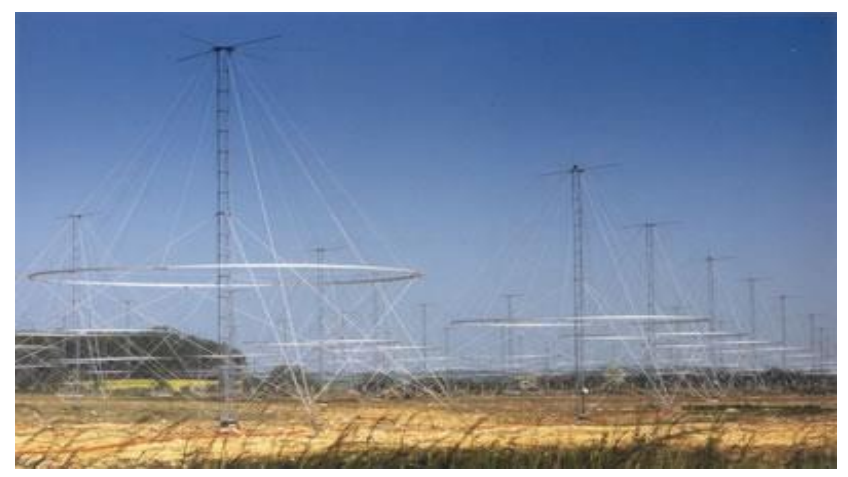

Fig. 1. Biconical antennas of a HF radar transmitting system operated by ONERA
In section II, the principle of measurement and the main constraints that apply to the proposed device are presented. In section III, the solution to the synchronization problem between the transmitter and receiver is explained. In section IV, the final system placed aboard the UAV is described. And finally, comparisons between the measured radiation pattern of a monopole antenna and a simulated one are commented in section $\mathrm{V}$.

\section{PRINCIPLE OF MEASUREMENT AND ASSOCIATED CONSTRAINTS}

To correctly characterize the near field of an antenna, it is required to map the three components $E_{\rho}, E_{\varphi}$ and $E_{z}$ of the near electric field, in amplitude and phase. This information must be known for different geographical points located on the surface of a virtual volume centered on the antenna as it can be seen in Fig. 2 .

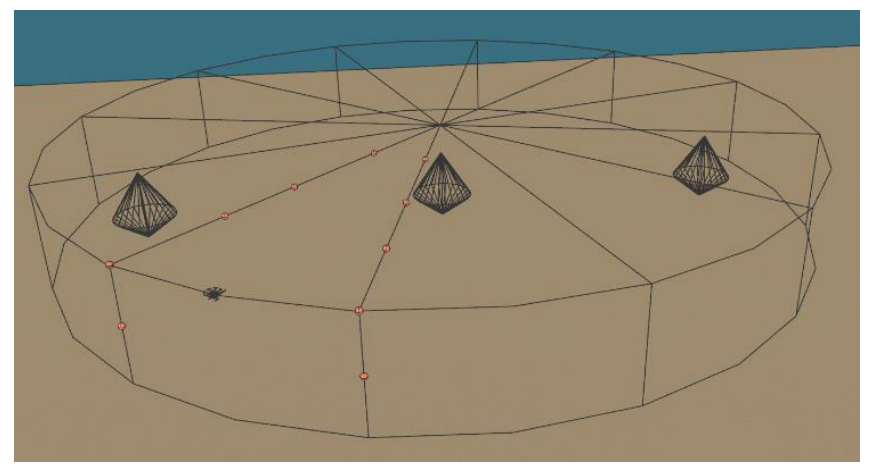

Fig. 2. Illustration of the measurement surface centered on the transmitting system composed by three biconical antennas

Similar measurement has already been achieved in the laboratory for higher frequency $(1 \mathrm{GHz})$ by applying the electromagnetic equivalence principle, using an electric near field probe named EFS-105 designed by Enprobe [3].

In order to achieve the near-field to far-field transformation previously described [2], it is imperative to quantify the electric field phase. It is therefore necessary, by some kind of synchronization, to estimate the phase difference between the antenna under test feed signal and the received signal. However, at $10 \mathrm{MHz}$, a precision on phase shift of $10^{\circ}$ corresponds to a precision of $3 \mathrm{~ns}$ on time shift. This issue is the major constraint for the development of the near-field measurement system.

Another critical issue is the precision needed for the near field probe positioning, in order to minimize the error induced on the calculated far-field. By assessing the robustness of the developed software at $1 \mathrm{GHz}$, we were able to determine that the needed precision was at least $\pm \frac{\lambda}{30}( \pm 1 \mathrm{~m}$ at $10 \mathrm{MHz})$. This 
matter was quickly resolved by integrating a GNSS RTK (Global Navigation Satellite System Real Time Kinematic) positioning system into the UAV. It provides an accuracy of less than $10 \mathrm{~cm}$ on the drone positioning. Finally, the use of a UAV imposes two other restrictions. First, the weight of the components needs to be optimized to reduce the impact on the drone autonomy. Last, it is necessary to move the electric field probe further apart from the drone in order to mitigate coupling effects between the carrier and the near field probe.

\section{SYNCRHONIZATION}

Performing a synchronization of the digitization clocks of the transmitted and received signals is a major challenge which is yet to be resolved [4]. Indeed, a nanosecond precision is difficult to achieve, even with a miniature atomic clock or with a GPSDO (Global Positioning System Disciplined Oscillator). That issue is related to the inevitable clock drift that affects the operating frequency of those tools as well as their sensitivity to vibrations, which are not negligible while using an UAV.

Therefore, it has been necessary to explore an alternative allowing to acquire the electric field phase without an equipment such as the one described above.

The chosen solution is to bring back, at ground level, the signal acquired by the near field probe, which is aboard the drone, by means of an optical fiber. This method has been concurrently developed by other research teams [5] and it allows us, by integrating an electro-optical converter on the UAV, to gather near electric field values, without digitizing the signal aboard the drone. Moreover, the optical fiber is not sensitive to radio frequency radiation and therefore resilient to any disturbances on the electric field information. At last, lightweight optical fiber has a low impact on the weight of the payload. This design makes possible to mimic the manipulation performed in laboratory and simplifies data processing. The next step has been to design the payload.

\section{PAYLOAD CONCEPTION}

The UAV used is a DJI Matrice 600 pro. This kind of industrial drone can lift as much as $6 \mathrm{~kg}$ for 15 minutes fly duration. The payload is mounted under the drone and it is necessary to comply with the integration restrictions in weight and size. To this end, an electro-optical converter has been modified in order to reduce its size and weight, so that it can be integrated inside the payload. For optimizing the payload weight, it has been decided to choose aluminum to build the platform hosting the different parts of the system. The payload, Fig.3, is made up of the electro-optical converter (1)), a $12 \mathrm{~V}$ battery (2) and the near field probe case housing (3)).

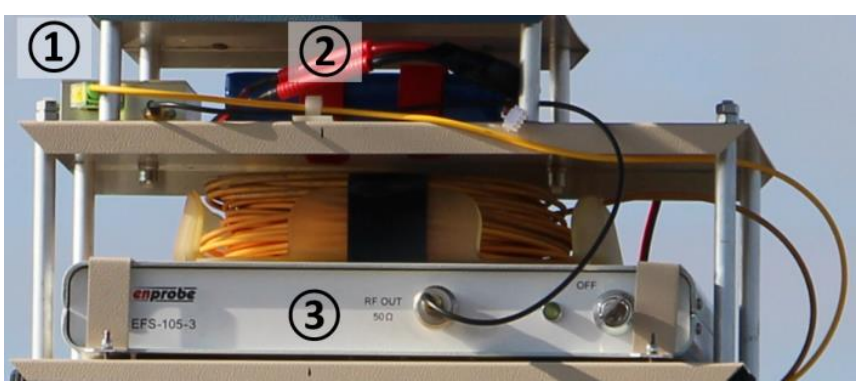

Fig. 3. Payload placed under the UAV
To mitigate coupling effects between the transmitting antennas and the drone, it is necessary to move the near field probe aside from the UAV. The electromagnetic simulation software CST (Computer Simulation Technology) has been used to estimate the needed distance between the sensor and the drone. Unfortunately, the drone manufacturer does not communicate on the properties of the UAV materials. Consequently the simulation has been performed assuming the worst-case scenario, which is that of a perfect electrical conductor (PEC) structure. In order to have a negligible error, it is required to locate the sensor at least at $70 \mathrm{~cm}$ from the UAV. For practical reasons due to landing constraints, the probe has to be placed on one of the sides of the drone. The required distance is obtained with a fiberglass tube as shown in Fig.4.

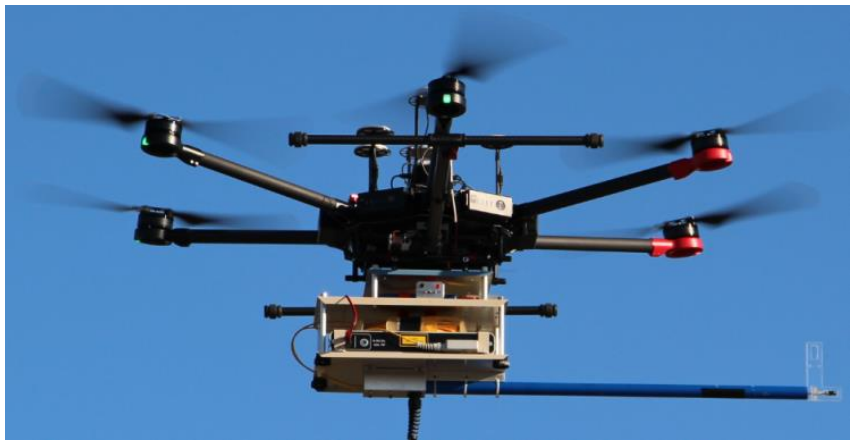

Fig. 4. DJI Matrice 600 pro equipped with the measurement system

The last part designed is the clamping device used to position the sensor in the three directions corresponding to the three electric field components. In order to simplify the measuring system and avoid adding extra weight, it has been decided not to use a remotely controllable motor that can change the orientation of the probe. The operation is therefore manually performed on ground. The drone positioning accuracy is about ten centimeters, so it can be considered that the UAV will stand at quasi-unchanged measurements points for the three flights. The clamping device, Fig.5, has been manufactured in polycarbonate.

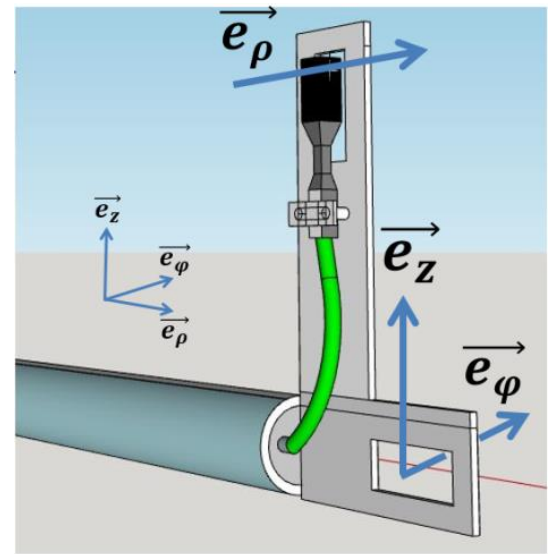

Fig. 5. Clamping device used to position the probe

\section{COMPARISONS BETWEEN MEASUREMENTS AND SIMULATIONS}

\section{A. Measurement setup}

In order to check the proper functioning of the abovedescribed system, it has been chosen to acquire the radiation pattern of a quarter-wave monopole antenna. This well-known 
kind of antennas enables to perform simple comparisons between measurements and simulations. A $6 \mathrm{~m}$ monopole antenna has been erected on a $20 \times 25 \mathrm{~m}^{2}$ ground plane. This ground plane was mounted above a concrete ground with unknown electrical parameters. The antenna impedance was matched to $50 \mathrm{ohms}$ at a frequency of $13.5 \mathrm{MHz}$ thanks to a RLC matching circuit. To avoid damages to the near field probe, which is in its saturation region for an electric field higher than $30 \mathrm{~V} / \mathrm{m}$, the antenna input power has been limited to $6 \mathrm{dBm}$. Feed and received signals have been both digitized with a NI PXIe-5171 oscilloscope that allows a 14-bit quantization and a $250 \mathrm{MS} / \mathrm{s}$ sample rate. Both signals have been digitized using the same clock, so the relative phase difference between the two signals is known. Measured antenna and associated ground plane can be seen in Fig.6.

Considering the radiation pattern of the monopole antenna as omnidirectional, it was not needed to measure the near electric field on a cylinder centered on the antenna. Indeed, by only measuring it on a radial line and duplicating the obtained data, it has been possible to generate a closed measurement cylinder around the antenna. GPS flight data have been recovered after each flight and have been used to determinate the position of the probe at any time.

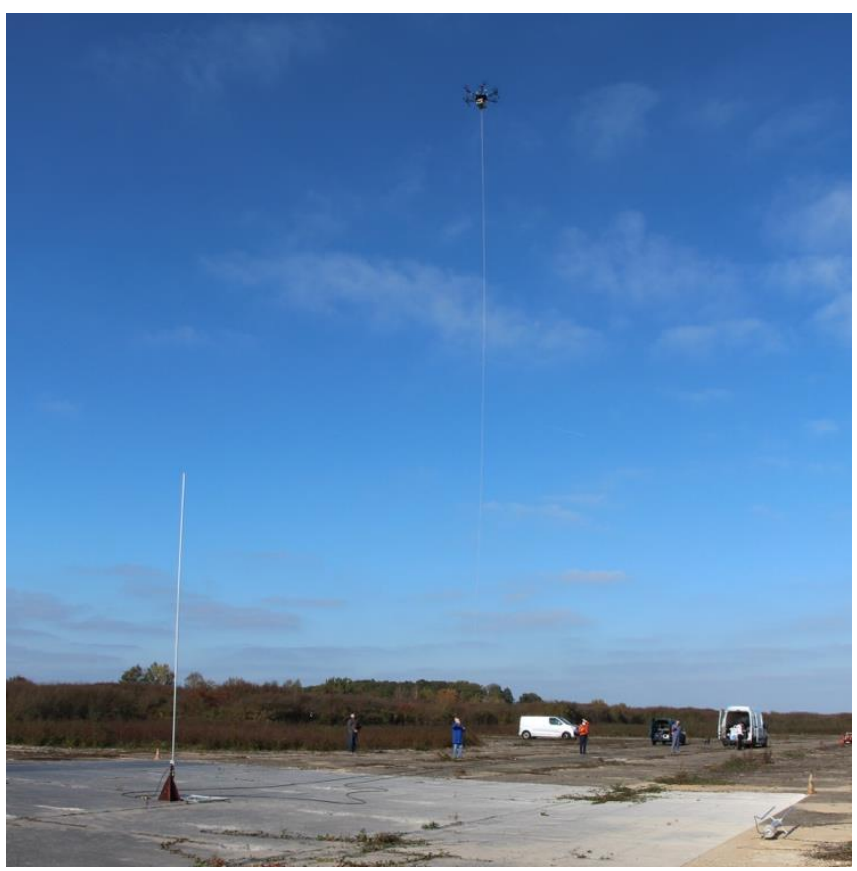

Fig. 6. Measured monopole antenna and associated ground plane

\section{B. Comparisons between measured and simulated near electric field}

The three electric field components have been measured. Since the near electric field $E_{\varphi}$ associated to a monopole antenna is close to zero, results are given only for $E_{\rho}$ and $E_{z}$ electric field components. As shown in Fig.7, two trajectories have been followed to measure $E_{\rho}$ and $E_{z}$. After neglecting slight movements in $y$ plane, comparisons with simulations have been performed.

CST Microwave and FEKO have been used to simulate near electric field radiated by the monopole antenna exhibited in figure 6. The antenna reflection coefficient $S_{11}$ was measured in order to know the exact radiated power. In consequence, comparisons between measurements and simulations have been made with absolute amplitude data.
Electrical delay, due to the coaxial cable and optical-fiber lengths, is not precisely known, so the field phase comparisons are achieved by shifting the measured phase with an arbitrary constant electrical delay. So, the differences between measured and simulated phases are interesting to compare. In addition, absolute field phase values are not needed to perform the near field to far field transformation with the previously developed software. Horizontal axis represents the total distance flown by the UAV. Zero corresponds to the position of the antenna. The first $25 \mathrm{~m}$ coincide with the displacement along the $x$-axis by the drone at a constant altitude. The last $9 \mathrm{~m}$ corresponds to the drone descent phase and landing.

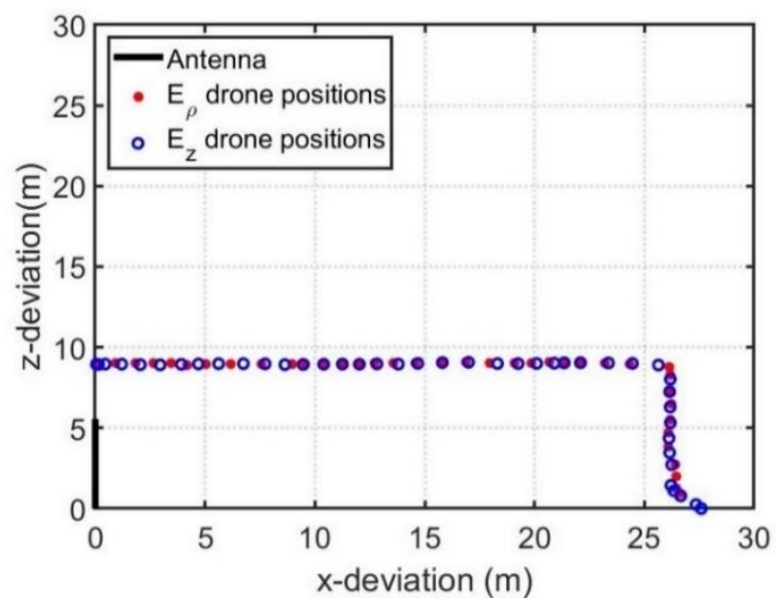

Fig. 7. Actual drone flight paths respectively used for measurements and simulations of $E_{\rho}$ and $E_{z}$
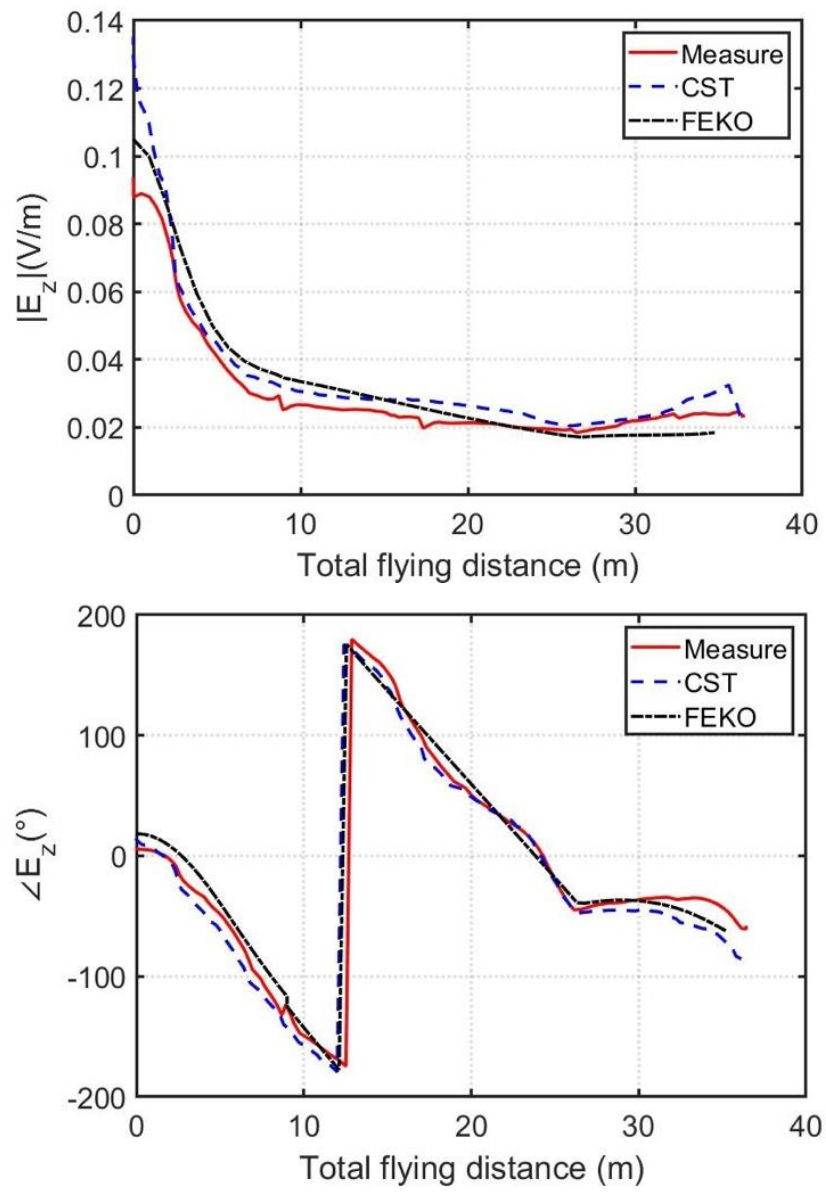

Fig. 8. Comparisons between measured and simulated near electric field $E_{z}$, in absolute amplitudes and relative phases 
Comparisons between measured and simulated $E_{z}$ data (amplitude and phase values) show a good agreement. However, there are some differences between the simulated data from CST and FEKO: that might be explained by the different electrical properties of the concrete used in simulation.
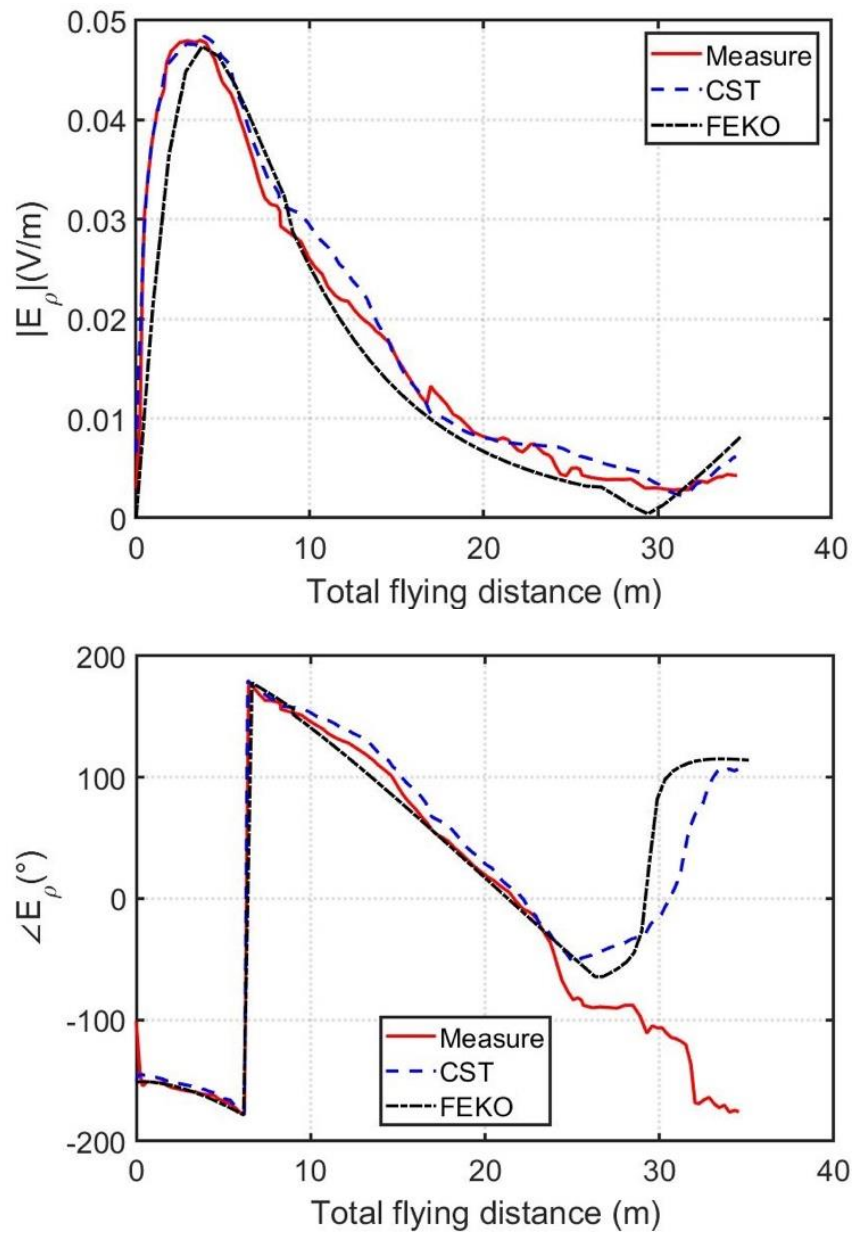

Fig. 9. Comparisons between measured and simulated near electric field $E_{\rho}$, in absolute amplitudes and relative phases

A considerable difference is shown on a part of the $E_{\rho}$ phase. It has not yet been elucidated, but it is of little importance. Indeed, the associated amplitude values are small and will have a low impact on the near-field to far-field transformation. Several successive measurements were made and the obtained results are similar. Near electric field measurements were satisfactory and reproducible, and so it has been possible to achieve a near field to far field transformation.

\section{Near field to far field transformation and comparisons}

The obtained far field radiation pattern has been compared to the simulated one computed using FEKO. Far field has been evaluated at $300 \mathrm{~m}$ from the monopole antenna. As it can be seen on figure 10, there is a good agreement between the two radiation patterns. In both cases, the maximum radiated power is at $\theta=50^{\circ}$. Differences between the two curves can be explained by the lack of knowledge about the properties of the concrete covering the ground on which the antenna is located. Those results confirm the relevance of carrying out a characterization of $\mathrm{HF}$ antennas with the proposed system. Successful measurements on a monopole operating at $50 \mathrm{MHz}$ were also performed and we look forward to test our measuring system on VHF array to determine the upper operating frequency of the system.

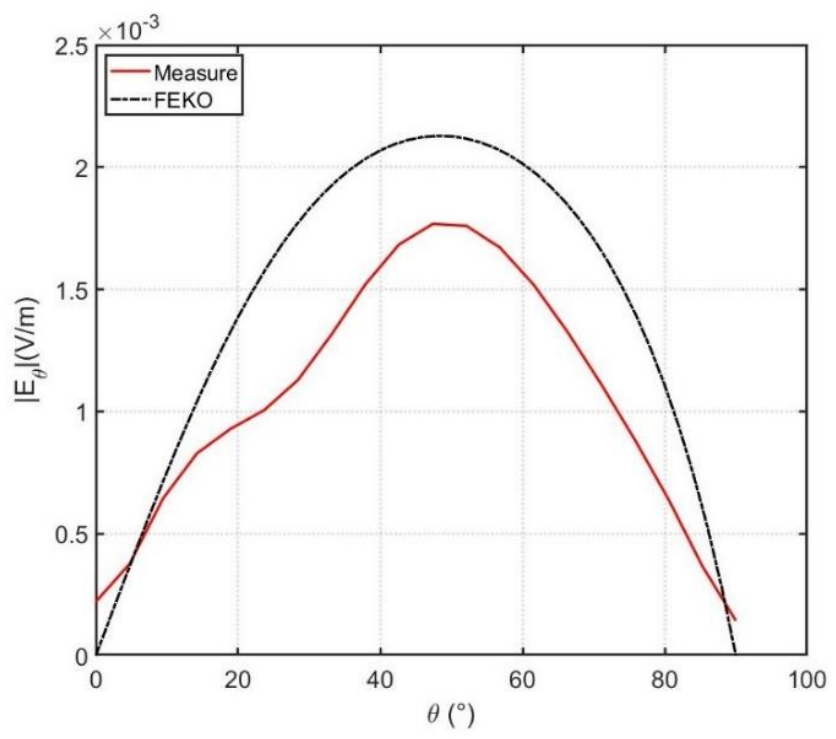

Fig. 10. Comparisons between measured and simulated far field evaluated at $300 \mathrm{~m}$ from the antenna

\section{CONCLUSION}

In this paper, a drone-embedded measuring system has been proposed, designed and tested. It is used to acquire the near electric field radiated by an antenna operating in the HF band. In order to perform the near-field to far-field transformation with a previously developed software, the amplitude and phase of the three components of the near electric field are required. The innovative solution here is to overcome the difficulty of synchronizing the clocks of transmission and reception systems, mounted aboard the UAV, by recovering the field values via an optical fiber. The constraints that apply to the design of the payload have been reviewed and the devices fulfilling them have been assembled and installed in the payload. Last, a successful test of the entire system has been carried out on an actual HF monopole antenna.

\section{ACKNOWLEDGMENT}

DGA (French Ministry of Defense) is thanked for its contractual support to the RAPID AC3M project for which the airborne system has been designed and developed.

\section{REFERENCES}

[1] R. H. Stehle, G. A. DesBrisay, A. L. Whitson, et G. H. Hagn, «RELEDOP: a full-scale antenna pattern measurement system for high-powered HF transmitting antennas », IEEE Trans. Broadcast., vol. $34, n^{\circ} 2$, p. $210-220$, june 1988.

[2] C. Djoma, M. Darces, et M. Hélier, «Prediction of Sky and Surface Wave Radiation of a Wideband HF Antenna », IEEE Antennas Wirel. Propag. Lett., vol. 14, p. 1149-1152, 2015.

[3] N. Bourey, M. Darces, et M. Hélier, «In Situ Antenna Far Field Estimation Based on Equivalent Sources », in 2018 USNC-URSI Radio Science Meeting (Joint with AP-S Symposium), 2018, p. 105-106.

[4] L. Orraca et al., « Compact high frequency receiver system for Arecibo observatory radiation pattern measurement », in 2018 IEEE Radio and Wireless Symposium (RWS), 2018, p. 10-13.

[5] T. Fritzel, R. Strauß, H. J. Steiner, C. Eisner, et T. Eibert, «Introduction into an UAV-based near-field system for in-situ and large-scale antenna measurements (Invited paper) », in 2016 IEEE Conference on Antenna Measurements Applications (CAMA), 2016, p. 1-3. 
